\title{
Analysis of Structure-Activity Relation for Primaquine Antimalarial Drugs by a Quantum Pharmacological Approach
}

\author{
S. K. Kokpol*, S. V. Hannongbua*, N. Thongrit*, S. Polman*, \\ B. M. RODE** and M. G. SCHWENDINGER** \\ *Computational Chemistry Unit Cell, Department of Chemistry, Faculty of Science, \\ Chulalongkorn University, Bangkok, Thailand \\ ** Department of Chemistry, Faculty of Science, University of Innsbruck, Innsbruck, Austria
}

\begin{abstract}
Linear models for the electronic structure-activity relations of primaquines based on molecular orbital calculations and pharmacological data observed in laboratory animals have been investigated. The optimized models seem to allow an evaluation of the antimalarial activity of primaquine drugs from calculated electron distributions of atoms while at the same time give strong indications about the active center of these and related types of antimalarial drugs.
\end{abstract}

Keywords Quantitative structure-activity relationship, active center, antimalarial drugs, molecular orbital calculations

Although a large number of studies concerning the quantitative structure-activity relationships (QSAR) of drugs have appeared during the past 20 years, only a few of them have made use of quantitative molecular orbital theory methods, e.g., in the case of diuretics ${ }^{1}$, barbiturates $^{2,3}$ and antibiotics. ${ }^{4-9}$ No such study has so far been published for antimalarial drugs.

In a previous study on linear modelling of the electronic structure-activity relations of chloroquine-type antimalarial drugs, based on semiempirical $\mathrm{CNDO} / 2$ MO SCF calculations ${ }^{10}$, it was shown that this approach can lead to useful model equations for the prediction of pharmacological activity from calculated atomic net charges, and that these equations allow conclusions about the relative importance of the compound's atoms for the activity. Therefore, this method is also a helpful tool in determining the structure and polarity of the active center of a drug.

In that study, evaluations of the possible relations of the atomic charges of all ring atoms and amino $\mathrm{N}$, dipole moment and ring charge with antimalarial activity $(A)$ in humans have led to a linear model containing $\mathrm{CNDO} / 2$ calculated atomic charges of $\mathrm{N1}$, $\mathrm{N} 2$ (amino), C4, C9 and $\mathrm{C} 10$ as parameters. Standard deviations could be improved by the inclusion of the dipole moment and ring charge, so that $\ln A$ could be predicted with an average accuracy of \pm 0.80 within a series of 17 chloroquine drugs.

In this work, a similar approach has been taken for other types of antimalarial drugs, namely primaquine and its substituted derivatives which have been investigated in numerous laboratory animal experiments with respect to their antimalarial activity. ${ }^{11}$

\section{Method}

For quantum chemical calculations, the previously used semiempirical CNDO/2 MO SCF method ${ }^{12}$ was again employed. The geometries of primaquine and its derivatives were taken from experimental data ${ }^{13}$, supported by standard assumptions whenevre necessary. The atomic electron densities and, thereby, net charges have been evaluated by Mulliken population analysis. ${ }^{14}$ Pharmacological activity data for antimalarial activity related to the standard quinine were taken from the literature"1 referring to chicken and duck as laboratory animals. Since pharmacological data strongly differ for both types of animals, two independent approaches for either series of data have been taken. In the case that more than one value for activity has been reported, the average of these values was used in our evaluation.

Following the approach in the case of chloroquines ${ }^{10}$, the linear model underlying the supposed electronic structure/activity relation is characterized by the equation

$$
\ln A=\Sigma P(i) q(i)+D
$$

where $A$ is the relative pharmacological acitivity in percent, $P(i)$ the parameter for the $i$ th atom, $q(i)$ the net charge of that atom (evaluated by subtracting the formal electron number of the atom from its calculated electron density in the compound) and $D$ the dipole 


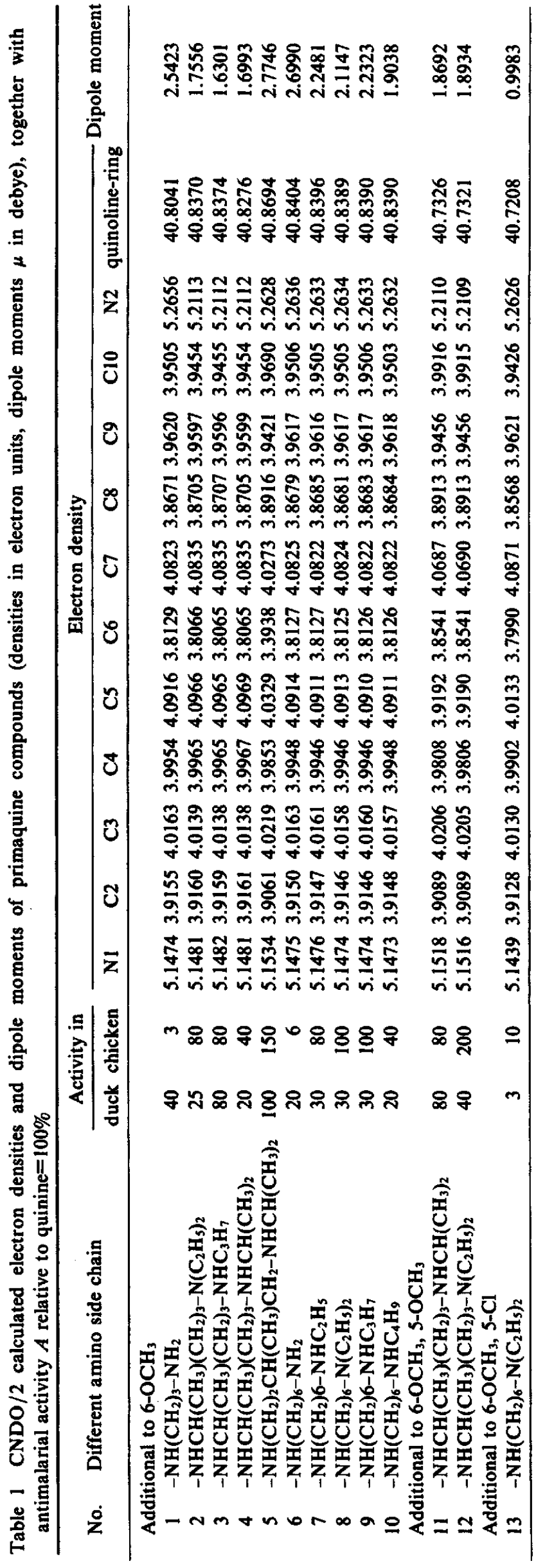

moment. As in our former investigation ${ }^{10}$, the model of Eq.(1) has been extended by including the over-all charge of the quinoline ring, $q$ (ring), and the calculated dipole moment $\mu$ of the compound, leading to the following equation:

$$
\ln A=P(i) q(i)+P(u) \mu+P(\text { ring }) q(\text { ring })+D .
$$

Searching for linear models for the type described in Eqs. (1) and (2), the relative importance of atoms in the molecule can gradually be recognized from the parameter size of these atoms. Thus, less important atoms can be excluded for the sake of a simpler model, allowing for a gradual evaluation of the structure of the active center of a drug.

\section{Results and Discussion}

The atomic electron densities and calculated dipole moments are listed in Table 1 for all compounds investigated, together with their reported pharmacological activities.

After some tentative model calculations, atoms N1, $\mathrm{N} 2, \mathrm{C} 7, \mathrm{C} 8, \mathrm{C} 9$ and $\mathrm{C10}$ (cf. Fig. 1) were shown to be the most sensitive centers for the drug's acitivity. Successive models were therefore tested, while, gradually including the charges at these centers and the ring charge and dipole moment of the additional quantity. The latter has been introduced simultaneously, since previous results had shown that their joint consideration seems to be advantageous. ${ }^{10}$

$$
\begin{array}{ll}
\text { Model } & \text { 1: N1, N2, C8, C9 } \\
\text { Model } & \text { 2: N1, N2, C8, C9, C7 } \\
\text { Model } & \text { 3: N1, N2, C8, C9, C7, C10 } \\
\text { Model } & \text { 4: N1, N2, C8, C9, C7, C10 q(ring), } \mu
\end{array}
$$

For all models parameters were fitted by linear regression; the quality of the model was then tested by 
evaluating the average deviation from the predicted value according to the observed activity, $c($ av.). Parameters and $c($ av.) values are listed in Table 2, supplemented by the value for the maximum deviation observed, $c$ (max.).

\section{Duck (Dataset a of Table 2)}

The smallest model has already been quite successful, as far as $c$ (av.) is concerned; however, the maximum observed deviation clearly indicates that some important factors have been neglected. The inclusion of $\mathbf{C 7}$ and $\mathrm{C10}$ shows, by the relative size of their parameters, that the charges of these atoms are highly relevant. Comparing the data of models 2 and 3 , the inclusion of both atoms seems to be essential. The data of model 3 for $c(a v$.) and $c$ (max.) are very satisfactory and acceptable, respectively. Further inclusion of $q$ (ring) and $\mu$ does not significantly improve this data. Model 3 , therefore, appears to represent the most reasonable approach and a good compromise between model simplicity and prediction accuracy.

\section{Chicken (Dataset b of Table 2)}

From the very beginning, linear modelling for chicken data proved to be more easy than for the data obtained in the case of duck. Already the most simple model leads to surprisingly low values for $c(\mathrm{av}$.) and $c$ (max.). In this case, the inclusion of $\mathrm{C} 7$ and $\mathrm{ClO}$ brings about only a slight improvement. Consideration of $q$ (ring) and $\mu$ is more efficient in this case. Still-due to the small number of pharmacological data-this could be a purely numerical effect of fitting. Thus, and in order to obtain equal model conditions for both series of data, we also favor model 3 for the case of chicken data.

A joint evaluation of model 3 for both data sets has led to the conclusion that the active center (i.e. the functional group whose electron distribution mostly influences the antimalarial activity of the compound) can be characterized by $\mathrm{N} 1, \mathrm{C} 8, \mathrm{C}, \mathrm{N} 2$ and - to a varying extent-C7 and $\mathrm{C} 10$. This finding sholuld be compared with the results derived from our previous study concerning chloroquine antimalarial activity in humans. ${ }^{10}$

These studies had indicated the active center of chloroquines to be formed by $\mathrm{N} 1, \mathrm{C4}, \mathrm{C} 9, \mathrm{Cl0}$ and $\mathrm{N} 2$; $\mathrm{C} 2$ and $\mathrm{C} 3$ have an additional, but minor influence ( $c f$. Fig. 2). Comparing both types of compounds, one can see that there are obvious similarities between both active centers as resulting from our evaluation of the electronic structure/activity relations:

- two nitrogens, one carrying a hydrogen atom, at a similar distance

- the nitrogens are linked by aromatic carbon atoms in a cis arrangement, by 2 and 3 atoms respectively

- the background of the active center is formed by bulky groups (aromatic ring system and side chain).

These common features allow some speculation about the potential receptor molecules for both drugs. The active center seems to be suitable for binding to nucleic bases, being a hydrogen bond acceptor via $\mathrm{N} 1$
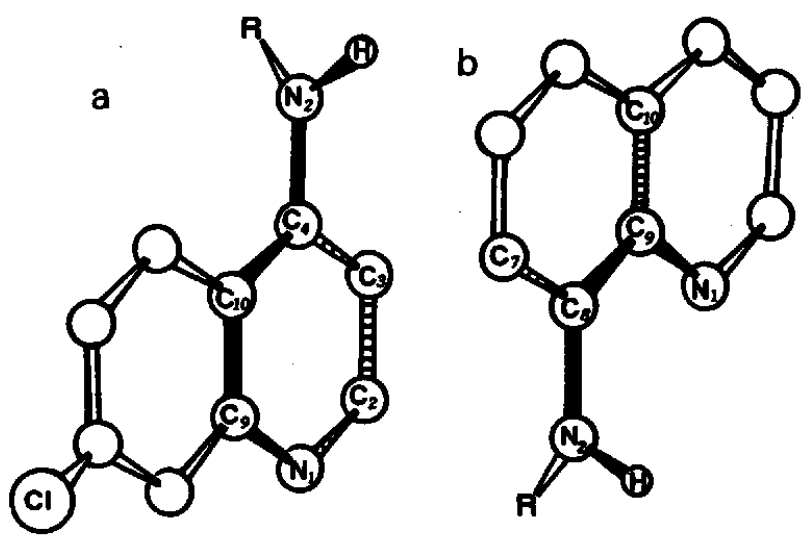

Fig. 2 Comparison of active centers according to quantum chemical calculations and linear modelling in chloroquine (a) and primaquine (b) antimalarial drugs.

Table 2 Parameters for linear models according to equations (1) and (2) and fitting characteristics $c($ av.) and $c($ max.) for pharmacological data a) of duck and b) of chicken

\begin{tabular}{|c|c|c|c|c|c|c|c|c|c|c|c|}
\hline \multirow{2}{*}{ Model } & \multicolumn{9}{|c|}{ Parameters } & \multirow{2}{*}{$c(\mathbf{a v})}$. & \multirow{2}{*}{$c(\max )}$. \\
\hline & N1 & N2 & $\mathrm{C8}$ & $\mathrm{C} 9$ & $\mathrm{C7}$ & $\mathrm{ClO}$ & ring & $\mu$ & $D$ & & \\
\hline $1 \mathrm{a}$ & 293.5 & -10.00 & -0.76 & 11.1 & - & - & 一 & - & -36.9 & 0.753 & 2.263 \\
\hline b & 647.6 & -0.06 & -0.86 & 128.2 & - & - & - & - & -87.2 & 0.267 & 0.811 \\
\hline $2 a$ & -3531.0 & -295.20 & 999.80 & 1929.0 & -670.4 & - & - & - & 862.6 & 0.698 & 1.859 \\
\hline b & 1822.0 & 87.00 & -308.10 & -460.8 & 205.9 & 一 & - & - & -363.4 & 0.247 & 0.710 \\
\hline $3 a$ & -2175.0 & -115.90 & 2128.00 & -1748.0 & 755.8 & -1531.00 & - & - & 430.5 & 0.353 & 1.166 \\
\hline b & 1815.0 & 86.00 & -314.20 & -441.0 & 198.2 & 8.23 & - & - & -361.1 & 0.244 & 0.713 \\
\hline $4 a$ & 152.9 & 24.30 & 1657.00 & -2273.0 & 986.7 & -1477.00 & -12.3 & -1.9 & -46.0 & 0.351 & 0.959 \\
\hline b & 4400.0 & 233.90 & -1023.00 & -617.8 & 391.0 & 257.60 & -3.7 & -2.1 & -877.0 & 0.162 & 0.471 \\
\hline
\end{tabular}


and a hydrogen bond donor via $\mathrm{N} 2-\mathrm{H}$. Once attached to a nucleic base, it would prevent the approach of other nucleic bases for pairing through its bulky background groups, provided the hydrogen bonding is strong enough. The compounds could be assumed, therefore, to interfere with the plasmodia's RNA binding to its nucleic bases and thus, hindering, replication. This assumption is backed by the fact that no antimalarial drug of either type is known with two substituents at $\mathrm{N} 2$ (and, hence, not providing a proton for hydrogen bonding).

This assumption would obviously suggest that the N2-H hydrogen charge is another parameter directly related to the drug's activity. As already mentioned in our previous study ${ }^{10}$, no attempt has been made to include this data into our models, due to the well-known poor description of hydrogen populations in the CNDO/2 framework. The results of this study, therefore, once more suggest an extension of the modelling to the more sophisticated and reliable $a b$ initio type of MO calculations, despite the larger number of methodical problems and the increased computational efforts emerging from such studies. ${ }^{10}$

The results of the present work confirm, however, that even semiempirical MO SCF calculations can be a very useful tool in designing potential new drugs and revealing a drug's active center, as already stated in our previous work. ${ }^{10}$ Quantum pharmacology, therefore, appears to have become another field of computational chemistry that can assist experimental research in a powerful and economic way.

Financial support by the Austrian Federal Ministry for Science and Research (GZ. 18889/7-10/87) and generous supply of computer time by the Computer Center of Chulalongkorn University are gratefully acknowledged.

\section{References}

1. Y. Shinagawa and Y. Shinagawa, Jpn. J. Pharmacol., 23, 615 (1973).

2. P. R. Andrews and G. P. Jones, Eur. J. Med. Chem., 16, 149 (1981).

3. F. Cara, A. Cardenas and R. Cetina, Rev. Lationam. Quim., 9, 4 (1978).

4. M. C. Manning and R. W. Woody, Biopolymers, 25, 2065 (1986).

5. B. J. Graves and D. B. Boyd, J. Antibiot., 37, 1642 (1984).

6. Y. G. Swyers and A. Hernandez-Laguna, J. Pharm. Sci., 72, 1011 (1983).

7. N. V. Joshi and V. S. R. Rao, J. Biol. Sci., 4, 209 (1982).

8. C. Petrolongo, G. Ranghino and R. Scordamaglia, Med. Chem. Adv., Proc. Intl. Symp., 7 (1980), Pergamon Press, Oxford (1981). Chem. Phys., 45, 279, 291 (1980).

9. D. B. Boyd, D. K. Herron, W. Lunn and W. Spitzer, J. Am. Chem. Soc., 102, 1812 (1980).

10. B. M. Rode, M. G. Schwendinger, S. U. Kokpol, S. V. Hannongbua and S. Polman, submitted for publication.

11. A. S. Alving, T. N. Pullman, B. Craige, Jr., R. Jones, Jr., C. M. Whorton and L. Eichelberger, J. Clin. Invest., 27, Supple., 35 (1947).

12. J. A. Pople, "Approximate Molecular Orbital Theory”, Mc. Graw-Hill, New York (1970).

13. L. E. Sutton, O. Kennard, A. A. Meconnell and A. D. Mitchel, "Tables of Interatomic Distance and Configuration in Molecules and Ions", The Chemical Society, London (1965).

14. R. S. Mulliken, J. Chem. Phys., 23, 1833, 1844 (1955); 36, 3428 (1962).

(Received August 10, 1988)

(Accepted October 24, 1988) 